\title{
Tests of Chiral Perturbation Theory with $\mathrm{K}_{\mathrm{e} 4}$ decays at CERN NA48/2 experiment *
}

\section{Spasimir Balev}

Physics Department, CERN, Geneva, Switzerland

E-mail: spasimir.balevecern.ch

\section{Brigitte Bloch-Devaux ${ }^{\dagger \ddagger}$}

Dipartimento di Fisica Sperimentale, Università degli Studi di Torino, Italy

E-mail: brigitte.bloch-devauxecern.ch

The NA48/2 collaboration has accumulated the largest samples to date of semi-leptonic charged kaon decays in the $\mathrm{K}_{\mathrm{e} 4}^{+-}\left(\mathrm{K}^{ \pm} \rightarrow \pi^{+} \pi^{-} \mathrm{e}^{ \pm} v\right)$ and $\mathrm{K}_{\mathrm{e} 4}^{00}\left(\mathrm{~K}^{ \pm} \rightarrow \pi^{0} \pi^{0} \mathrm{e}^{ \pm} v\right)$ modes. From 1.1 million $\mathrm{K}_{\mathrm{e} 4}^{+-}$decays, form factors in the S- and P-wave have been extensively studied. Branching ratio and form factors have been measured at unprecedented precision. From $\sim 66000 \mathrm{~K}_{\mathrm{e} 4}^{00}$ decays, preliminary values of the Branching ratio and form factor have been obtained at a percent level precision. The comparison of Branching ratio and form factor values in both $\mathrm{K}_{\mathrm{e} 4}$ modes sheds new light on isospin symmetry breaking effects. Form factor measurements are major inputs to the study of low energy QCD and are powerful tests of Chiral Perturbation Theory predictions.

The European Physical Society Conference on High Energy Physics

18-24 July, 2013

Stockholm, Sweden

${ }^{*}$ This report is dedicated to the memory of our colleague and friend S. Balev who passed away much too early on September 8, 2013. This contribution owes a lot to his strong implication in the analysis.

$\dagger$ Speaker.

$\ddagger$ On behalf of the NA48/2 collaboration: Cambridge, CERN, Chicago, Dubna, Edinburgh, Ferrara, Florence, Mainz, Northwestern, Perugia, Pisa, Saclay, Siegen, Turin, Vienna 


\section{The $\mathrm{K}_{\mathrm{e} 4}$ decay formalism}

Four-body final state decays are described by five kinematic variables, namely for $\mathrm{K}_{\mathrm{e} 4}$ decays, the Cabibbo-Maksymowicz variables [1]]: two invariant masses $S_{\pi}=M_{\pi \pi}^{2}$ and $S_{e}=M_{e v}^{2}$ and three angles $\vartheta_{\pi}, \vartheta_{e}$ and $\varphi$. The hadronic current is described by form factors which can be developed in a partial wave expansion as suggested in [2]. Limiting the expansion to S- and P-waves and considering a unique phase $\delta_{p}$ for all $\mathrm{P}$-wave form factors, two axial $(F, G)$ and one vector $(H)$ complex form factors contribute to the transition amplitude: $F=F_{s} e^{i \delta_{s}}+F_{p} e^{i \delta_{p}} \cos \theta_{\pi}, G=$ $G_{p} e^{i \delta_{p}}, H=H_{p} e^{i \delta_{p}}$. From the differential rate study in the 5-dimensional space, four real form factors $\left(F_{s}, F_{p}, G_{p}\right.$ and $\left.H_{p}\right)$ and a single phase difference $\left(\delta=\delta_{s}-\delta_{p}\right)$ are then measured, together with their energy variation with $S_{\pi}$ and $S_{e}$. In the neutral pion mode $\mathrm{K}_{\mathrm{e} 4}^{00}$, the variables $\vartheta_{\pi}$ and $\varphi$ are irrelevant and the form factors reduce to the single $F_{s}$ value due to Bose statistics.

\section{Beam and detector setup}

Two simultaneous $\mathrm{K}^{ \pm}$beams were produced by $400 \mathrm{GeV} / c$ protons from the CERN/SPS impinging on a beryllium target. Opposite charge particles with a central momentum of $(60 \pm$ 3) $\mathrm{GeV} / c$ were selected and focused $\sim 200 \mathrm{~m}$ downstream at the first spectrometer chamber. A magnetic spectrometer (a dipole magnet surrounded by two sets of drift chambers) measured the momentum $(p)$ of charged decay products with a relative precision of $\sim 1 \%$ for $10 \mathrm{GeV} / c \mathrm{mo}-$ mentum tracks. It was followed by a scintillator hodoscope consisting of two planes segmented into horizontal and vertical strips achieving a very good $\sim 150 \mathrm{ps}$ time resolution. A liquid krypton calorimeter ( $\mathrm{LKr}$ ), 27 radiation length thick, was used to measure electromagnetic deposits $(E)$ and to identify electrons through their $E / p$ ratio (the energy and transverse position resolutions were $\sim 1 \%$ and $\sim 1.5 \mathrm{~mm}$ (resp.) for $10 \mathrm{GeV}$ showers). A two-level trigger logic selected and flagged events with a high efficiency for both $\mathrm{K}_{\mathrm{e} 4}$ topologies. A detailed description is available in [3].

\section{Event reconstruction, analysis procedure and results}

Event reconstruction and selection follow similar paths for both signal modes: the $\mathrm{K}_{\mathrm{e} 4}^{+-}\left(\mathrm{K}_{\mathrm{e} 4}^{00}\right)$ candidates are reconstructed from three charged tracks (one track and four photons forming two $\left.\pi^{0} \mathrm{~s}\right)$ pointing to the same decay vertex. The abundant $\mathrm{K}_{3 \pi}$ decay modes $\left(\mathrm{K}^{ \pm} \rightarrow \pi^{+} \pi^{-} \pi^{ \pm}\right.$and $\mathrm{K}^{ \pm} \rightarrow \pi^{0} \pi^{0} \pi^{ \pm}$) are considered as normalization modes. They are recorded concurrently with the same trigger logic and subjected to the same reconstruction as the signal events. Kinematic separation of signal and normalization candidates is obtained by requiring (or not) missing mass and missing transverse momentum in the $\mathrm{K}_{3 \pi}$ hypothesis. Extra requirements of electron identification $(0.9<E / p<1.1$ and properties of LKr associated shower consistent with the electron hypothesis) ensure a low background contamination of order $\sim 1 \%$ relative. The remaining background is mainly due to $\mathrm{K}_{3 \pi}$ decays with a $\pi^{ \pm}$faking an electron response in $\mathrm{LKr}$ or followed by the rare $\pi \rightarrow e v$ decay, while accidental coincidence with another track/photon is one order of magnitude lower. Most background contributions are measured from control data samples. Geometrical acceptances for the four decay modes are obtained from a MC simulation including detector geometry, material description and local imperfections. They are typically $\sim 18-20 \%$ in the $\mathrm{K}_{\mathrm{e} 4}^{+-}$and $\mathrm{K}_{3 \pi}^{+-}$modes and $2 \%(4 \%)$ in the $\mathrm{K}_{\mathrm{e} 4}^{00}\left(\mathrm{~K}_{3 \pi}^{00}\right)$ modes. 
Form factors values are obtained by adjusting the differential distributions of simulated signal candidates to those of data candidates in small boxes of the multi-dimensional kinematical space. Branching ratios (BR) are obtained as:

$$
\mathrm{BR}\left(\mathrm{K}_{\mathrm{e} 4}\right)=\left(N_{s}-N_{b}\right) / N_{n} \cdot\left(A_{n} \varepsilon_{n}\right) /\left(A_{s} \varepsilon_{s}\right) \cdot \mathrm{BR}\left(\mathrm{K}_{3 \pi}\right),
$$

where $N_{s}$ is the number of signal candidates $\left(1.1 \times 10^{6} \mathrm{~K}_{\mathrm{e} 4}^{+-}, 6.6 \times 10^{4} \mathrm{~K}_{\mathrm{e} 4}^{00}\right), N_{b}$ the background contribution (10552 for $\mathrm{K}_{\mathrm{e} 4}^{+-}, 708$ for $\left.\mathrm{K}_{\mathrm{e} 4}^{00}\right)$ and $N_{n}$ the number of normalization events $(19 \times$ $\left.10^{8} \mathrm{~K}_{3 \pi}^{+-}, 94 \times 10^{6} \mathrm{~K}_{3 \pi}^{00}\right) . A_{s}\left(A_{n}\right)$ are the geometrical acceptances described above and $\varepsilon_{s}\left(\varepsilon_{n}\right)$ are the trigger efficiencies, well above $95 \%$ and similar for signal and normalization modes. $\operatorname{BR}\left(\mathrm{K}_{3 \pi}\right)$ are the normalization Branching ratios. The detailed $\mathrm{K}_{\mathrm{e} 4}^{+-}$form factor and $\mathrm{BR}$ measurements are already available in [ $₫$ ] while the corresponding $\mathrm{K}_{\mathrm{e} 4}^{00}$ measurements are preliminary.

Branching ratios, inclusive of $\mathrm{K}_{\mathrm{e} 4 \gamma}$ decays, are obtained at improved precision:

$$
\begin{aligned}
\mathrm{BR}\left(\mathrm{K}_{\mathrm{e} 4}^{+-}\right) & =\left(4.257 \pm 0.004_{\text {stat }} \pm 0.016_{\text {syst }} \pm 0.031_{\text {ext }}\right) \times 10^{-5}, \mathrm{PDG} \text { value }=(4.09 \pm 0.10) \times 10^{-5}, \\
\mathrm{BR}\left(\mathrm{K}_{\mathrm{e} 4}^{00}\right) & =\left(2.585 \pm 0.010_{\text {stat }} \pm 0.010_{\text {syst }} \pm 0.032_{\text {ext }}\right) \times 10^{-5}, \mathrm{PDG} \text { value }=(2.2 \pm 0.4) \times 10^{-5},
\end{aligned}
$$

where systematic errors include uncertainties on acceptance, resolution, beam geometry, particle identification, trigger efficiencies and radiative corrections. External errors stem from the normalization mode BR uncertainties and are now the dominant errors. The $F_{s}$ form factor variations with $q^{2}\left(=S_{\pi} / 4 m_{\pi^{+}}^{2}-1\right)$ are displayed in Fig.1. The same quadratic behavior is present in both modes for $\left(q^{2}>0\right)$ and a deficit of events is observed below the $2 m_{\pi^{+}}$threshold $\left(q^{2}=0\right)$ in the $\mathrm{K}_{\mathrm{e} 4}^{00}$ mode as observed in the $\mathrm{K}_{3 \pi}^{00}$ mode [阿]. It can be explained by final state charge exchange scattering $\left(\pi^{+} \pi^{-} \rightarrow \pi^{0} \pi^{0}\right)$. The absolute form factors have also been obtained as:

$$
\begin{aligned}
F_{S}\left(\mathrm{~K}_{\mathrm{e} 4}^{+-}\right) & =5.705 \pm 0.003_{\text {stat }} \pm 0.017_{\text {syst }} \pm 0.031_{\text {ext }}, \\
F_{S}\left(\mathrm{~K}_{\mathrm{e} 4}^{00}\right) & =6.092 \pm 0.012_{\text {stat }} \pm 0.017_{\text {syst }} \pm 0.045_{\text {ext }} .
\end{aligned}
$$

The observed tension between $F_{s}\left(\mathrm{~K}_{\mathrm{e} 4}^{+-}\right)$and $F_{s}\left(\mathrm{~K}_{\mathrm{e} 4}^{00}\right)$ as well as between $\mathrm{BR}\left(\mathrm{K}_{\mathrm{e} 4}^{+-}\right)$and $2 \mathrm{BR}\left(\mathrm{K}_{\mathrm{e} 4}^{00}\right)$ points to possible isospin symmetry breaking, radiative or other effects to be investigated.
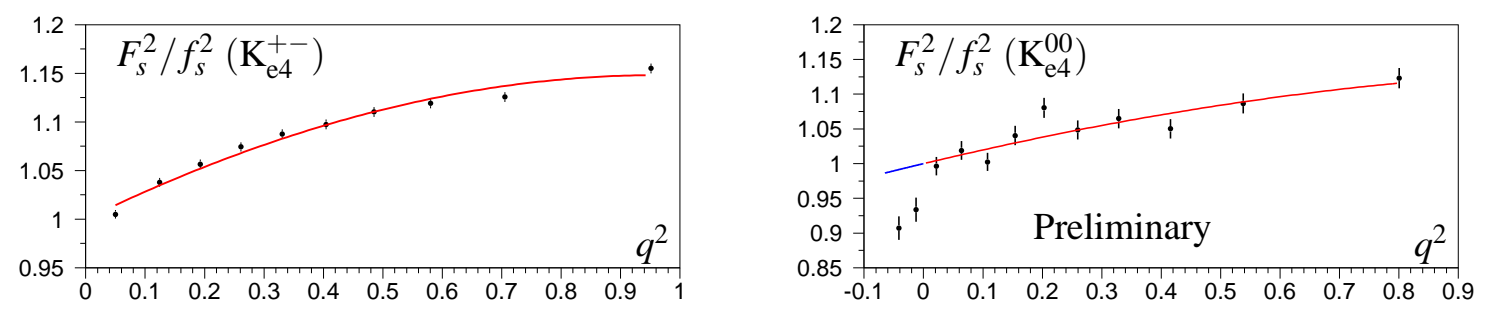

Figure 1: Relative $F_{s}^{2} / f_{s}^{2}$ form factor measurements function of $q^{2}$ in the $\mathrm{K}_{\mathrm{e} 4}^{+-}$(Left) and the $\mathrm{K}_{\mathrm{e} 4}^{00}$ (Right) mode. By construction $F_{s}^{2} / f_{s}^{2}\left(q^{2}=0\right)=1$. Red lines are degree-2 polynomial fits to the data (stat. errors only) for $q^{2}>0$ while the blue line is the extrapolation to negative values.

\section{References}

[1] N. Cabibbo and A. Maksymowicz, Phys. Rev. B438, (1965) 137.

[2] A. Pais and S. Treiman, Phys. Rev. 168, (1968) 1858.

[3] V. Fanti et al, Nucl. Instrum. Methods A 574, (2007) 433.

[4] J. Batley et al, Eur. Phys. J. C 70, (2010) 635, ibidem, Phys. Lett. B 715, (2012) 105.

[5] J. Batley et al, Eur. Phys. J. C 64, (2009) 589. 